\title{
Entre promotion et prévention
}

Louise Potvin est Professeure titulaire au Département de médecine sociale et préventive à l'Université de Montréal, titulaire de la Chaire Approches communautaires et inégalités de santé et Associate Editor de SPM

Les quatre articles originaux qui composent ce numéro de SPM illustrent bien la difficulté d'établir des démarcations précises et univoques entre les interventions dites de promotion de la santé et celles qui affichent la prévention comme objectif. Nous publions en effet deux articles reçus en réponse à notre appel de 2005 pour solliciter des articles portant sur la promotion (Kloek et al. 2006 (p. 259-272); Märki et al. 2006 (p. 273-280)). Il serait cependant bien difficile d'identifier un ou quelques critères de distinction entre ces deux articles et les deux autres qui portent respectivement sur les résultats de recherche qui supportent les recommandations d'avoir une activité physique modérée pour une période de 30 minutes à tous les jours (Bucksch \& Schlicht 2006 (p. 281-301) et sur l'effet de réduction des inégalités en matière de caries dentaires associé à la fluoration de l'eau de consommation par les municipalités brésiliennes (Peres et al. 2006 (p. 302-310)). Mais au fait qu'est-ce qui distingue la prévention de la promotion?

En Occident la plupart des grands documents de présentation de la santé publique caractérisent la prévention des maladies et la promotion de la santé comme deux des fonctions essentielles de la santé publique. Par exemple, dans le «Programme national de santé publique 2003-2012» du Québec, on stipule que «la promotion de la santé et du bienêtre inclut l'ensemble des actions qui visent à influencer les déterminants de la santé de façon à permettre aux individus, aux groupes et aux communautés d'avoir une plus grande emprise sur leur santé par l'amélioration de leurs conditions et leurs modes de vie» (Santé et des services sociaux Québec 2002). Un peu plus loin, il est dit que «la prévention englobe des activités qui visent avant tout à réduire les facteurs de risque associés aux maladies, aux problèmes psychosociaux et aux traumatismes ainsi qu'à détecter les signes hâtifs des problèmes de santé ou des problèmes psychosociaux.» Alors qu'il est clair que dans les deux cas l'on vise à agir en amont des problèmes de santé, la distinction que l'on fait entre les déterminants de la santé dans le cas de la promotion et les facteurs de risque dans le cas de la prévention est beaucoup plus nébuleuse. La promotion d'un mode de vie sain qui inclut au moins une période de 30 minutes d'une activité physique modérée relève-t-elle seulement de la promotion? Une telle habitude de vie ne contribue-t-elle pas spécifiquement à la diminution du risque de plusieurs maladies comme le diabète de type 2 et les maladies cardio-vasculaires. La fluoration de l'eau potable qui contribue à réduire les inégalités de santé bucco-dentaire entre les riches et les pauvres n'est-elle vraiment que de la prévention de la carie dentaire? Une telle intervention ne participe-t-elle pas à influencer l'un des déterminants importants de la santé, soit la capacité de s'alimenter par une dentition saine? Il me semble que la collection d'articles proposés dans ce numéro permet d'élargir la réflexion sur la fonction et le rôle de la santé publique dans nos sociétés modernes audelà de ses fonctions et modalités d'action. Pour John Last la santé publique est l'ensemble des connaissances, pratiques et valeurs mobilisées pour le maintien et l'amélioration de la santé de tous les individus d'une population. C'est une responsabilité et un effort collectif pour la protection, la promotion et la restauration de la santé dans une population par des actions organisées (Last 1998). Qu'il s'agisse de prévention ou de promotion il me semble que l'objectif doit être le même, soit de participer à l'effort de santé publique. En fait, les quatre articles qui forment ce numéro illustrent bien l'interdépendance de deux objectifs poursuivis par la santé publique à travers des programmes nationaux, notamment au Canada, aux États-unis et en Suède, soit l'amélioration de la santé de la population ET la diminution des écarts de santé entre différents segments de la population. En effet, le seul objectif d'amélioration de la santé de la population, même s'il est essentiel ne garantit pas à lui seul une diminu- 
tion des disparités et un accroissement de la justice sociale (Advisory Committee on Population Health and Health Safety 2005). En ce sens les questions importantes ne sont pas de savoir si nos actions sont de l'ordre de la prévention ou de la promotion de la santé, mais plutôt d'examiner comment ces actions participent soit à l'amélioration de la santé dans une population, soit à la diminution des écarts, ou aux deux.

Louise Potvin

\section{Références}

Advisory Committee on Population Health and Health Safety (ACPHHS) (2005). Réduire les disparités sur le plan de la santé - Rôles du secteur de la santé : document de travail. Ottawa: Ministère de la santé.

www.phac-aspc.gc.ca/ph-sp/disparities/pdf06/ disparities_discussion_paper_f.pdf

Bucksch J, Schlicht W (2006). Health-enhancing physical activity and the prevention of chronic diseases - An epidemiological review. Soz Praventiv Med 51(5): 281-301.

Kloek GC, van Lenthe FJ, Mertens YMG, Koelen MA, Mackenbach JP (2006). Process Evaluation of a Dutch Community Intervention to Improve Health Related Behaviour in Deprived Neighbourhoods. Soz Praventiv Med 51(5): 259-272.
Last JM (1998). Public health and human ecology. $2^{\text {nd }}$ edition. Stamford, Connecticut: Apllaton $\&$ Lange.

Märki A, Bauer GF, Nigg CR, Conca-Zeller A, Gehring TM (2006). Transtheoretical Model based exercise counselling for older adults in Switzerland: quantitative results over a 1-year period. Soz Praventiv Med 51(5): 273-280.

Peres MA, Fereira Antunes JL, Glazer Peres K (2006). Is water fluoridation effective in reducing inequalities in dental caries distribution in developing countries? Soz Praventiv Med 51(5): 302-310.

Santé et services sociaux Québec (2002). Programme national de santé publique 2003-2012. Québec: Ministère de la santé et des services sociaux. http://publications.msss.gouv.qc.ca/acrobat/f/ documentation/2002/02-216-01.pdf

\section{Address for correspondence}

Prof. Louise Potvin, PhD GRIS-Université de Montréal P.O.Box 6128, Station centre-ville Monréal, QC H3C 3J7

Canada

Tél.: +1 5143436142

Fax: +1 5143432207

e-mail: louise.potvin@UMontreal.CA

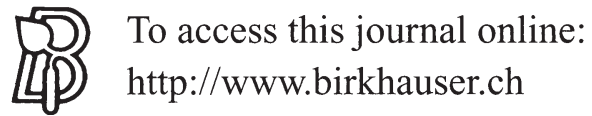

